\title{
Cinco Dimensiones Claves para Avanzar en la Inclusión Educativa en Latinoamérica
}

\section{Five Key Dimensions to Move to Inclusive Education in Latin America}

\begin{abstract}
Universidad Complutense de Madrid, España
El artículo destaca los principales avances y los desafíos sociales y educativos en los países latinoamericanos y plantea que las exigencias del siglo XXI no deben olvidar la agenda pendiente del siglo Xx. En este contexto, se formulan cinco prioridades políticas, tanto sociales como educativas, que pueden ayudar a dar un mayor impulso a la inclusión social y educativa, contribuyendo a articular estas dos agendas: los acuerdos políticos, la prioridad de la infancia, el fortalecimiento y desarrollo de la profesión docente, la transformación cultural y el cambio en las actitudes. Se concluye que en estos procesos de transformación de la sociedad y del sistema educativo se encuentran los principales desafíos y resalta la necesidad de un acuerdo político y social. De esta forma se proporcionará un impulso sostenido a lo largo de los próximos años para avanzar hacia la educación inclusiva.
\end{abstract}

Álvaro Marchesi *

Laura Hernández

Descriptores: Latinoamérica; Políticas educacionales; Diversidad; Cultura; Transformación social.

\begin{abstract}
The article highlights the advances and social and educational challenges in Latin American countries and points out that the demands of the 21 st century should not forget the outstanding agenda of the 20th century. In this context, five political priorities, both social and educational, are formulated that would promote social and educational inclusion, contributing to articulate these two agendas: political agreements, priority of childhood, strengthening and development of the teaching profession, cultural transformation and change in attitudes. It is concluded that in these processes of transformation of society and the educational system are the main challenges and highlights the need for a political and social agreement. This will provide a sustained impulse over the next few years to move towards inclusive education.
\end{abstract}

Keywords: Latin America; Educational policies; Diversity; Culture; Social transformation.

\section{Introducción}

La Conferencia Mundial sobre necesidades educativas especiales celebrada hace ahora 25 años en Salamanca (España) fue un encuentro excepcional tanto por la participación masiva de representantes de los países de todos los continentes, como por el impacto a lo largo del tiempo de su Declaración final y de su Marco Acción. Es muy difícil encontrar en el ámbito educativo un proyecto de cambio que haya sido tan escuchado y que haya servido de referencia a tantas políticas públicas.

*Contacto: amarches@psi.ucm.es

ISSN: 0718-7378

www.rinace.net/rlei/
Recibido: $\quad 15 / 07 / 19$

$1^{\text {a }}$ Evaluación: 20/08/19

Aceptado: $\quad$ 13/09/19 
Ahora bien, estas afirmaciones no deben hacernos creer que un encuentro y un acuerdo de estas características provoca un impulso transformador que conduce a alcanzar de forma casi inmediata gran parte de las metas señaladas. Desgraciadamente, como la realidad no se cansa de manifestar, la situación suele ser muy diferente. La Conferencia Mundial de Salamanca marcó unos objetivos ambiciosos, pero es preciso posteriormente avanzar hacia ellos teniendo en cuenta las condiciones económicas, sociales y educativas, los recursos disponibles y el compromiso colectivo para realizar los cambios necesarios. En estos procesos de transformación de la sociedad y del sistema educativo se encuentran los principales desafíos.

Es justo reconocer que se han producido en América Latina avances importantes en la educación y en la respuesta a la diversidad que desde los centros y las aulas se proporciona a los estudiantes. Sin embargo, todavía se mantienen retos antiguos enormemente importantes y otros han aparecido por el impacto de los cambios sociales y culturales más recientes. La agenda del siglo XX no ha terminado aún en los países latinoamericanos, que deben enfrentarse al mismo tiempo a la agenda del siglo XXI (Schwartzman y Cox, 2009). Ello supone un esfuerzo enorme que obliga a acelerar el ritmo de los cambios.

El presente artículo se plantea principalmente destacar las cinco prioridades políticas, sociales y educativas que contribuirían a articular ambas agendas: los acuerdos políticos, la prioridad de la infancia, el fortalecimiento de los docentes, la transformación cultural y el cambio en las actitudes. De esta forma, se proporcionará un impulso sostenido a lo largo de los próximos años para avanzar en la dirección de la educación inclusiva.

\section{Los progresos y los retos en la sociedad y en la educación latinoamericana}

Entre los cambios positivos que se han producido en la sociedad y en la educación de los países latinoamericanos, quizás el más representativo sea la reducción de la pobreza, reflejo de las profundas desigualdades que afectan a toda la región. Desde el año 2002 hasta el 2017, el porcentaje de personas con ingresos por debajo de la línea de la pobreza ha descendido del $45 \%$ al 30\% (CEPAL, 2018). Pero el hecho de que todavía el $30 \%$ de los ciudadanos latinoamericanos vivan en esta situación, manifiesta claramente las dificultades vitales de una parte significativa de la población, lo que afecta a sus posibilidades de alcanzar un mínimo básico de bienestar y de garantizar buenas condiciones para el desarrollo y la educación de sus hijos.

Esta misma dicotomía entre los avances significativos y los graves retos pendientes está presente también en toda la educación. El acceso a la educación es el logro más relevante alcanzado en las dos últimas décadas debido al incremento del gasto público y a la mayor importancia otorgada a la educación. La tasa de escolarización en educación primaria en 2013 alcanza el $94 \%$, mientras que en secundaria supera ese mismo año el $76 \%$, veinte puntos más que en 1995 (OREALC-UNESCO, 2013)

Sin embargo, continúa existiendo graves carencias en los recursos existentes en las escuelas. Duarte, Guargiulo y Moreno (2012) muestran que el 40\% de las escuelas de educación básica no disponen de biblioteca, que el 35\% no tiene espacios para el deporte y que el $11 \%$ no tienen acceso a la electricidad. 
Limitaciones importantes se encuentran también en la situación de los docentes, aunque las diferencias entre los países latinoamericanos son muy importantes. Su formación presenta bastantes carencias, sus salarios son reducidos si se les compara con profesionales de un nivel similar, las condiciones de trabajo son complicadas en muchas escuelas y el tiempo de aprendizaje de los alumnos insuficiente (Cabrol y Székely, 2012).

No es extraño por las razones anteriormente apuntadas que un porcentaje importante de los alumnos latinoamericanos muestren retrasos en sus aprendizajes. Los nueve países latinoamericanos participantes en PISA 2015 (OCDE, 2018) obtienen resultados por debajo del promedio de la OCDE en todas las pruebas.

\section{Los desafíos de la inclusión educativa}

Estas insuficiencias afectan especialmente a aquellos estudiantes que tienen más dificultades para mantenerse en el sistema educativo de forma continuada, pues sus demandas educativas tienen más riesgo de no poder ser atendidas ni por sus familias ni por sus escuelas.

Conviene recordar que los sistemas educativos deben garantizar dos derechos a los alumnos con discapacidad y otros considerados con necesidades educativas especiales: el derecho a aprender de acuerdo con sus posibilidades y el derecho a aprender en común con sus compañeros de similares edades. La mayor dificultad se encuentra en que las escuelas con escasos recursos y con un nivel de calidad insatisfactorio tienen serias dificultades de garantizarlos. No es suficiente que estos estudiantes estén con sus compañeros. Es preciso al mismo tiempo asegurar las mejores condiciones para que aprendan lo más posible.

¿Qué sucede en los países latinoamericanos? Los datos obtenidos por las instituciones internacionales son muy escasos, a menudo fragmentarios y con diferencias entre los países sobre los criterios para determinar las características de un alumno con discapacidad. El estudio más reciente ha sido realizado por el Instituto de Estadística de la UNESCO (2018), que analiza y compara datos de 49 países, de los cuales 11 son latinoamericanos en cinco indicadores referidos al acceso, la permanencia, el abandono escolar y el analfabetismo adulto entre 2006 y 2015.

En general, los estudios realizados muestran algunas tendencias generales: existen importantes diferencias entre los países en la educación de los alumnos con discapacidad o con otras necesidades educativas especiales; ha habido un continuo progreso en el acceso de los alumnos con discapacidad al sistema educativo, aunque suelen permanecer en él menos tiempo que sus compañeros sin especiales dificultades; los compromisos internacionales y los cambios en la legislación han provocado un lento pero continuado avance en la inclusión educativa, si bien las condiciones sociales y educativas dificultan un progreso más acelerado.

\section{Cinco dimensiones claves para el progreso educativo y el avance en la inclusión}

Hay que reconocer que las urgencias educativas en los países latinoamericanos son profundas y afectan a todas las etapas del sistema educativo, desde la educación infantil 
hasta la secundaria, la formación técnico profesional y la inserción laboral de los jóvenes. Al mismo tiempo, la mejora de las condiciones de las escuelas y de los profesores junto con otros factores relacionados con la calidad educativa como el liderazgo escolar, la supervisión y la evaluación educativa, las políticas de equidad y las escuelas de tiempo completo son otros tantos retos que exigen atención y nuevos recursos. Sin embargo, los resultados de las investigaciones educativas y la evidencia empírica más consistente, así como el análisis de las políticas públicas de éxito, ponen de manifiesto que determinados factores son especialmente importantes para avanzar en una educación de calidad que sea al mismo tiempo inclusiva: los acuerdos políticos y sociales, la extensión de una educación infantil equitativa e inclusiva de calidad, el fortalecimiento de la profesión docente y el cambio en la cultura y en las actitudes hacia la inclusión social y educativa.

\subsection{Acuerdos políticos y sociales}

Las transformaciones sociales y educativas que son necesarias para impulsar procesos de inclusión de calidad exigen no solo capacidad de analizar la situación actual y las prioridades más importantes, sino un esfuerzo firme, sostenido y creciente al menos a lo largo de la próxima década. Para ello, es necesario un acuerdo político y social que permita mantener estas prioridades, aunque existan cambios en la presidencia de los países y en sus gobiernos.

Mucho se ha hablado y escrito sobre los pactos en el ámbito educativo. El interés por la estabilidad en el ámbito educativo y por evitar continuos cambios ha sido una de las razones esgrimidas para convencer sobre su necesidad. Sin embargo, a pesar de sus posibles ventajas, escasos acuerdos políticos se han concretado en los países latinoamericanos. Posiblemente, ha influido en ello las desconfianzas entre los partidos políticos y la voluntad de conseguir un acuerdo lo más amplio posible lo que, en gran medida, de forma paradójica, ha sido una de las causas de su paralización.

Por estas razones, lo que ahora se plantea en estas líneas es un acuerdo sobre las prioridades fundamentales en la política educativa, en las políticas sociales y en los mensajes públicos para favorecer un proceso inclusivo de calidad que asegure su continuidad. El acuerdo que se propone es en gran medida educativo, pero también social, en la medida en que los valores y las creencias culturales, así como las actitudes ante la diversidad tienen una enorme influencia en las posibilidades de progreso de la inclusión educativa. Un acuerdo cuyos elementos claves se exponen a continuación.

\subsection{La ampliación y la equidad de la educación infantil}

Prácticamente todas las investigaciones concluyen en que los primeros años de vida de los niños y niñas son fundamentales para su desarrollo y sus aprendizajes posteriores. Los programas que favorecen el cuidado de los niños, su alimentación, el intercambio comunicativo y lingüístico, las relaciones sociales, el interés por los objetos, la exploración y el descubrimiento son importantes para todos los niños, pero más especialmente para aquellos que viven en situaciones de pobreza, en desventaja social o manifiestan necesidades educativas especiales (Peisner-Feinberg, 2007). La inversión en una educación temprana centrada en los niños y en su desarrollo con educadores preparados y con condiciones educativas favorables es la inversión económica más rentable por los beneficios que produce en los niños en sus primeros años y por la ayuda que les supone para enfrentarse a los retos futuros (Rolnick y Grunewald, 2003). 
Las investigaciones neurocientíficas (Center of the Developing Child at Harvard University, 2016) han comprobado que la protección de la infancia sobre todo en sus primeros años es mucho más beneficiosa que las intervenciones posteriores para superar posibles problemas futuros. Este cuidado debe orientarse al desarrollo de las dimensiones cognitivas, comunicativas, lingüísticas, emocionales y sociales, así como a apoyar la salud y la nutrición de los niños y de sus madres. El desarrollo y la educación integral de la infancia en contextos estables y seguros, que facilitan las interacciones comunicativas y sociales de los adultos con los niños y entre los niños y que les animan a explorar y a descubrir son una garantía para su bienestar futuro y para su incorporación en el entorno social y comunitario. Por ello, es necesario incrementar la oferta de educación infantil de forma significativa, con educadores bien formados, que reciban una retribución económica satisfactoria y que tengan las mejores condiciones para realizar bien su trabajo, es decir, para poder dedicarse a favorecer el desarrollo y el aprendizaje de todos y cada uno de los niños/as que están bajo su responsabilidad.

La ampliación y la mejora de la calidad y equidad de la educación infantil debe incorporar también el apoyo y la formación a las familias para el cuidado de sus hijos. Si los primeros años de la vida del niño son tan importantes y los niños y niñas pasan la mayoría de su tiempo con sus familias, es necesario ampliar el foco de la educación infantil al conjunto del entorno familiar. Ello supone que los educadores dediquen tiempo a la orientación de las familias y que promuevan prácticas inclusivas entre los niños y entre sus familias. De esta forma, se sentarán las bases no solo para un mejor desarrollo de los niños con discapacidad o necesidades educativas especiales, sino para el desarrollo de actitudes positivas de toda la comunidad educativa hacia la inclusión.

Además, sería importante implicar a diferentes instituciones públicas y privadas en el apoyo a los derechos de la infancia y en ofrecer respuestas coordinadas a las demandas sociales y educativas de los niños y de las niñas. La participación de los municipios es imprescindible para avanzar en la construcción de ciudades educadoras e inclusivas, abiertas a la diversidad y al encuentro cívico y comprometidas con mejorar las condiciones de vida y de educación de todos los niños y las niñas.

Sin embargo, la situación actual es todavía insuficiente. El gasto público en educación infantil y preprimaria como porcentaje del PIB en los países latinoamericanos y caribeños es muy inferior al de otras etapas educativas: $0,4 \%$ frente al 1,9\% en educación primaria, $1,4 \%$ en educación secundaria y 0,6\% en educación terciaria no universitaria (Busso et al., 2017). Además, los maestros y educadores que trabajan en la educación infantil tienen menos formación, salarios más bajos y peores condiciones de trabajo (Cabrol y Szèkely, 2012).

Un acuerdo que incluyera la ampliación de la educación infantil, el acceso de los grupos sociales en situación de mayor desigualdad, la mejora de sus condiciones para elevar su nivel de calidad, el compromiso de diferentes instituciones para garantizar los derechos de la infancia y abrir mejores posibilidades para ella y la prioridad de los niños y niñas en situación de desigualdad, entre ellos con discapacidad para acceder a los programas existentes proporcionaría un enorme respaldo a las políticas de inclusión educativa.

\subsection{El fortalecimiento de la profesión docente}

Una de las competencias principales de los maestros, posiblemente la más complejas, es la respuesta educativa a la diversidad de los alumnos. En la medida en que el objetivo de la 
inclusión no es solo mantener a los estudiantes en el aula con sus compañeros, sino facilitar al máximo sus posibilidades de aprendizaje, es preciso que el docente sea capaz de diseñar actividades que favorezcan estos aprendizajes. Buena parte de ellas podrían desarrollarse en colaboración con sus compañeros, aunque otras, dependiendo de las condiciones del estudiante, podrían realizarse de forma individual o con otros compañeros que compartan situaciones similares.

No cabe duda de que la calidad de la respuesta educativa a la diversidad de los alumnos depende en gran medida del número de alumnos por aula, de los maestros de apoyo presentes en el centro y de los recursos disponibles. Pero hay que reconocer que el factor principal para el éxito de la inclusión educativa en el aula se encuentra en las competencias del profesor en este ámbito, en su sensibilidad hacia las necesidades de los alumnos y en su compromiso con la inclusión educativa.

Estas consideraciones destacan la importancia de otorgar un especial valor a las competencias de los docentes en su desarrollo profesional para favorecer la inclusión educativa. En primer lugar, en su formación inicial. No basta con que exista en el currículo alguna materia o créditos sobre la educación inclusiva o sobre la educación de los alumnos con necesidades educativas especiales. Es preciso que la respuesta a la diversidad de los estudiantes esté incorporada en todas las materias de estudio. El objetivo es que los futuros profesores sean conscientes de que enseñar matemáticas, lengua, ciencias sociales o música exige tener en cuenta la diversidad de los alumnos y que han de ser capaces de planificar su enseñanza y su evaluación y diseñar sus actividades sabiendo que la diversidad será la norma en sus aulas, mientras que la homogeneidad será excepcional.

Esta misma orientación debe formar parte de la formación permanente de los docentes. Ahora los profesores han acumulado experiencia y se han enfrentado a los retos que supone la educación inclusiva. Por ello, los modelos de formación que se organizan en torno a la reflexión de los docentes de la misma escuela o de escuelas con proyectos similares suelen tener una mayor incidencia para mejorar la enseñanza (Marchesi y Martín, 2014).

Es importante no olvidar las condiciones en las que los profesores desarrollan su trabajo. Los proyectos inclusivos, sobre todo si se realizan en entornos sociales desfavorecidos, son muy exigentes y necesitan un cuidado especial. El apoyo de las administraciones educativas debería concretarse en un mayor tiempo disponible para preparar las clases, para coordinarse con otros docentes y para colaborar con las familias. Además, sus recursos disponibles deberían ser más numerosos. Tratar a todas las escuelas de la misma manera sin tener en cuenta su diversidad y los diferentes retos a los que se enfrentan supone aplicar un principio de homogeneidad que es ajeno a la realidad educativa.

También la respuesta a la diversidad debería formar parte de la evaluación de las escuelas. En el modelo propuesto por Marchesi y Pérez (2019), se incluye una dimensión relativa a la inclusión educativa junto con las condiciones del centro, el contexto sociocultural, los procesos de centros y de aula, la convivencia y los resultados. Sus indicadores principales son la presencia de una cultura inclusiva en la escuela, la existencia de una respuesta educativa adecuada a la diversidad de los alumnos, la coordinación entre los profesionales para ofrecer una respuesta educativa satisfactoria a los alumnos que experimentan dificultades y la adaptación de los procedimientos de evaluación a estos alumnos. 
Finalmente, el compromiso de los docentes con la inclusión educativa debería tenerse en cuenta en la valoración de sus méritos y en su desarrollo profesional. Marchesi y Pérez (2018) han formulado un modelo de evaluación para el desarrollo profesional de los docentes basado en la valoración de sus competencias que incluye entre ellas la capacidad de responder de forma flexible a la diversidad de sus alumnos. Sus principales indicadores son coordinarse con otros profesionales para responder a las diferentes demandas educativas de los alumnos, tener en cuenta en la programación a los alumnos con dificultades o de otras culturas, diseñar situaciones de aprendizaje para responder a la diversidad de los alumnos en el aula y adaptar a ellos los procedimientos de evaluación.

Situar al profesorado en el centro de las políticas educativas a favor de la inclusión no supone un matiz añadido. Es más bien un enfoque integral que deriva de la consideración de que una educación de calidad debe ser equitativa e inclusiva, por lo que el fortalecimiento de la profesión docente supone necesariamente mejorar sus condiciones y sus prácticas en beneficio de la inclusión.

\subsection{El valor de la cultura}

La inclusión educativa depende en gran medida del convencimiento de la mayoría de la sociedad de que la convivencia y el aprendizaje con alumnos diversos es positivo para todo el alumnado. Cuando el otro distinto es percibido como un peligro para la convivencia o el progreso educativo, se levanta y refuerza una de las principales barreras para la inclusión. La importancia que revela la dimensión cultural, en la que se incluyen también las creencias y las actitudes, aconseja prestarle una especial atención.

Somos lo que somos en gran medida por la cultura que hemos aprendido. En la medida en que los diferentes grupos humanos desarrollan distintas normas, valores, símbolos y tradiciones, existe una gran diversidad cultural, por lo que se puede afirmar que la diversidad forma parte del significado más genuino de cultura. Además, la cultura no es estática, sino que cambia de manera continua, en gran medida por las relaciones entre diferentes grupos. Por ello, la intervención en el ámbito cultural para favorecer el reconocimiento y la diversidad humana tiene una gran importancia para avanzar en la inclusión educativa.

En el fondo, las relaciones entre diferentes grupos reflejan la visión personal y colectiva de los que supone el Yo frente a los Otros diferentes. El cambio fundamental que debe producirse para el desarrollo de genuinas actitudes inclusivas es que se suprima la distancia entre el yo, expresión de un grupo, y el otro, que forma parte de un colectivo diferente, y se transforme en el "nosotros", donde unos y otros son vividos como miembros del mismo grupo a pesar de las diferencias.

En relación con los colectivos con discapacidad, la visión del otro puede convertirse en el reflejo de lo que podríamos ser nosotros mismos, o, desde otra perspectiva, en la interpelación a una actitud solidaria y transformadora que nos obligaría a modificar buena parte de las prioridades institucionales, sociales y personales. Las personas con discapacidad pueden despertar actitudes más acogedoras y solidarias (Hernández, 2015) que los colectivos inmigrantes o de culturas minoritarias. Los otros, personas con discapacidad, tienden a ser percibidos como miembros del propio grupo social y cultural y, por tanto, como parte del "nosotros", aunque se manifiestan diferencias notables entre los miembros de cada de estos grupos. 
Hay un elemento en el campo de la cultura que es especialmente importante: los mapas de significado. A través de ellos nos relacionamos con los demás, con los que consideramos de los "nuestros" o de los "otros", y marcan nuestras preferencias, nuestras señas de identidad y nuestros modelos ideales.

Las actitudes hacia los otros diferentes se manifiestan en distintas formas de relación (expulsión, segregación, asimilación o inclusión), en el lenguaje y en la comunicación. Lenguaje y conocimiento se estructuran mutuamente y reflejan nuestra visión del "nosotros" y de los "otros". El lenguaje, además, articula las relaciones con los demás y transmite la percepción que nuestro grupo de referencia tiene sobre los demás grupos culturales o sociales.

Estos modelos de representación social y sus correlatos discursivos se aprenden a través de las conversaciones, los ejemplos, los comentarios, las noticias, las películas, los medios de comunicación y un sinfín de actividades cotidianas vividas en la familia, en las escuelas y en las calles. De este modo, los niños van construyendo sus propias actitudes. No todos, quizás, pero sí la mayoría, presionados por la exigencia de su entorno hacia la conformidad social. Como afirma Van Dijk (2007), las estructuras discursivas afectan a la mente de las personas, por lo que el discurso racista favorece la reproducción de los prejuicios y estereotipos étnicos y la discriminación de los otros.

Los cambios impulsados en las últimas décadas a favor de denominaciones que sustituyan a las etiquetas y estereotipos negativos son expresión del valor que se le otorga al lenguaje para comprender a las personas y a los colectivos diferentes. Conviene recordar que etiquetar a una persona supone la utilización de un descriptor específico para referirse a ella (Baglieri y Shapiro, 2012). Inevitablemente, la etiqueta tiende a poner en primer plano y a generalizar esa característica, lo que conlleva a una visión distorsionada de la realidad. Las etiquetas conducen a los estereotipos, o son su expresión lingüística, y facilitan la categorización de las personas y de los colectivos así designados. El proceso puede terminar con la descalificación de los individuos y de los grupos y, como consecuencia, con la estigmatización social: determinados rasgos generan una profunda desaprobación social.

El desarrollo cultural se imbrica con el desarrollo individual y está en el origen de las transformaciones mentales. Desde esta perspectiva, no es posible comprender el desarrollo de las actitudes de los niños hacia los diferentes colectivos si no se incorpora el análisis de la historia, del contexto y de la cultura. La intervención en el ámbito cultural para favorecer la inclusión se extiende de forma natural al campo de las actitudes.

\subsection{Cambios en las actitudes sociales y educativas}

La importancia de las actitudes hacia la inclusión educativa fue recogida en Declaración de Salamanca (UNESCO, 1994). En ella se establece que los responsables políticos, los directores de escuela y los maestros deben promover actitudes positivas hacia los alumnos con necesidades educativas especiales, ya que las escuelas ordinarias con esta orientación integradora representan el medio más eficaz para combatir las actitudes discriminatorias, crear comunidades de acogida, construir una sociedad integradora y lograr la educación para todos.

La tesis más generalizada es que la actitud incluye tres elementos básicos interrelacionados: creencias o componente cognitivo, un componente emocional o afectivo y una acción o comportamiento. Por ello, las escuelas que cuidan especialmente la 
dimensión emocional y moral de sus alumnos son las que tienden a promover una actitud más positiva hacia la inclusión educativa.

Las actitudes no son innatas ni aparecen de forma súbita, sino que son aprendidas gradualmente a través de la experiencia. La manera en la que el niño se socializa, el grupo social de referencia y las pautas de socialización son determinantes destacados de las actitudes que va construyendo a lo largo de su evolución.

Conforme el niño se desarrolla, empieza a categorizar al resto de las personas con precisión creciente, ampliando progresivamente las variables que utiliza para esta clasificación, incluyendo aspectos salientes como la etnia o la complexión física, u otros menos perceptivos como la clase social o la nacionalidad. La adquisición de estas categorías vendrá determinada por la importancia que la sociedad atribuye a los diferentes grupos o características (Brown, 1995). De ahí la gran influencia de la sociedad en el desarrollo de las actitudes hacia las diferencias en el ámbito educativo. La cultura social inclusiva es, por tanto, un poderoso factor para el establecimiento de actitudes inclusivas positivas en el ámbito educativo.

Respecto a las personas con discapacidad, las actitudes de las personas que les rodean pueden suponer obstáculos más importantes para la inclusión en la comunidad que los derivados de la propia situación de discapacidad (Siperstein et al., 2003).

En la última década, un número creciente de estudios ha abordado la dimensión social de la educación inclusiva. Aunque un alumno con discapacidad esté físicamente en la escuela ordinaria, ello no conlleva que se logre su inclusión social, es decir, que establezca redes sociales y de amistad con los compañeros. Como indican Flores, Aguado y Alcedo (2009), el éxito de la inclusión de los niños con discapacidad depende tanto de las actitudes de los compañeros y de los profesores como de la existencia de un currículo bien adaptado. Nowicki y Sandieson (2002) sugieren incluso que la actitud de los estudiantes hacia las personas con discapacidad es uno de los principales problemas de la educación inclusiva y que las actitudes negativas de los iguales son una barrera para el progreso de la educación inclusiva.

De Boer (2012) realizó una exhaustiva revisión de los estudios publicados desde 1998 hasta 2011 sobre las actitudes de los estudiantes hacia los compañeros con discapacidad. La autora concluye que la mayoría de las investigaciones analizadas muestran que los alumnos tienen creencias, e intenciones de comportamiento neutras hacia sus compañeros con discapacidad. Argumenta que los resultados neutros no son motivo de especial preocupación, ya que por lo menos no son negativos, pero concluye que se debe tener presente que ello implica que en las diferentes muestras se encuentran estudiantes que poseen actitudes muy positivas y estudiantes con actitudes muy negativas. Esto último dificultaría de manera muy significativa la inclusión del alumno con discapacidad.

Junto con las actitudes de los estudiantes hacia la diversidad, es necesario subrayar nuevamente la importancia crucial de las actitudes de los profesores, pues son ellos los que pueden influir en las actitudes de los estudiantes y los que pueden impulsar un modelo de enseñanza y aprendizaje sensible y adaptado a la diversidad de sus estudiantes. Además, la actitud del profesorado va a repercutir en las expectativas del docente sobre el desarrollo y el aprendizaje de sus alumnos.

Hay que señalar finalmente que las actitudes de las familias ocupan también un papel importante. Determinadas expresiones, palabras o gestos, así como la forma de manifestar 
su visión de la diversidad en sus prácticas cotidianas promueven una determinada orientación en la formación de actitudes en los niños. No cabe duda de que las familias que son recelosas de la educación inclusiva pueden influir negativamente en las actitudes y el comportamiento de sus hijos e hijas.

El valor de la cultura y de las actitudes positivas en el avance de la inclusión educativa refuerza también los otros tres factores que se han expuesto en este trabajo: la importancia de que la educación inclusiva se amplíe y fortalezca desde los primeros años; el apoyo a los docentes, así como su formación y su sensibilidad hacia la diversidad de los alumnos; y la necesidad de un acuerdo político y social que no solo se oriente a mejorar la calidad de las escuelas desde una perspectiva inclusiva, sino que también aborde el cambio cultural necesario para que la comprensión, la valoración y la garantía de los derechos de todas las personas sin discriminación formen parte de los valores y creencias mayoritarios en la sociedad.

\section{Referencias}

Baglieri, S. y Shapiro, A. (2012). Disabilities studies and the inclusive classroom. Critical practices for creating least restrictive attitudes. Nueva York, NY: Routledge.

Brown, R. (1995). Prejudice: Its social psychology. Oxford: Blackwell.

Busso, M., Cristia J., Hincapié D., Messina J. y Ripani. L. (2017). Aprender mejor. Políticas públicas para el desarrollo de habilidades. Washington, DC: Banco Interamericano de Desarrollo (BID).

Cabrol, M. y Székely, M. (2012). Educación para la transformación. Washington, DC: Banco Interamericano de Desarrollo (BID).

Center of the Developing Child at Harvard University. (2016). From best practices to breakthrough impacts. A science-based approach to building a more promising future for young children and families. Cambridge, MA: Harvard University.

CEPAL. (2018). Panorama social de América Latina 2018. Informe anual. Santiago de Chile: CEPAL.

De Boer, B. (2012). Inclusion: A question of attitudes? A study on those directly involved in the primary education of students with special educational needs and their social participation. Groninger: Stichting Kinderstudies.

Duarte, J., Gargiulo C. y Moreno M. (2012). Infraestructura escolar y aprendizajes en la educación básica latinoamericana: un análisis a partir del SERCE. En M. Cabrol y M. Székely (Eds.), Educación para la transformación (pp. 205-244). Washington D.C.: Banco Interamericano de Desarrollo (BID).

Flores, M. A., Aguado, A. y Alcedo, M. A. (2009). Revisión y análisis de los programas de cambio de actitudes hacia personas con discapacidad. Anuario de Psicología Clínica y de la Salud, 5, 85-98.

Hernández, L. (2015). Estudio sobre las actitudes de los niños de 4 a 8 años hacia las diferencias étnicoculturales y hacia la discapacidad en Colombia, Guatemala y España (Tesis doctoral). Universidad Complutense de Madrid.

Marchesi, A. (2019). Salamanca 1994-2019: There is still a long way to Latin America. International Journal of Inclusive Education, 23(7), 841-848.

https://doi.org/10.1080/13603116.2019.1622803 
Marchesi, A. y Pérez. E. M. (2018). Modelo de evaluación para el desarrollo profesional docente. Madrid: Fundación SM e IDEA.

Marchesi, A. y Pérez, E. M. (2019. Modelo de evaluación de centros docentes para mejorar la educación. Madrid: Fundación SM e IDEA.

Nowicki, E. A. y Sandieson, R. (2002). A meta-analysis of school-age children's attitudes towards persons with physical or intellectual disabilities. International Journal of Disability, Development and Education, 49(3), 243-265.

http://dx.doi.org/10.1080/1034912022000007270

OCDE. (2018). Competencias en Iberoamérica: Análisis de PISA 2015. Madrid: OECD-Fundación Santillana.

OREALC-UNESCO. (2013). Situación educativa de América Latina y el Caribe: Hacia la educación de calidad para todos al 2015. Santiago de Chile: OREALC-UNESCO.

Peisner-Feinberg, E. S. (2007). Los jardines infantiles y su impacto en el desarrollo de los niños. En J. Bennett (Ed.), Cuidado infantil-educación y cuidado en la primera infancia. Enciclopedia sobre el desarrollo de la primera infancia (pp. 1-6). Montreal: Centre of Excellence for Early Childhood Development (CEECD).

Rolnick, A. y Grunewald, R. (2003). Early childhood development with a high public return. Fedgazette, 14, 7-23.

Schwartzman, S. y Cox. C. (2009). Las agendas pendientes de la educación. En S. Schwartzman y C. Cox (Eds.), Políticas educativas y cohesión social en América Latina (pp. 9-29). Santiago de Chile: Uqbar Editores.

Siperstein, G. N., Norins, J., Corbin, S. y Shriver, T. (2003). Multinational study of attitudes toward individuals with intellectual disabilities. Washington, DC: Special Olympics.

Van Dijk, T. A. (2007). Racismo y discurso en América Latina. Barcelona: Gedisa.

\section{Breve CV de los autores}

\section{Álvaro Marchesi}

Catedrático emérito de Psicología Evolutiva y de la Educación en la Universidad Complutense de Madrid. Desempeñó el cargo de Secretario General de la Organización de Estados Iberoamericanos para la Educación, la Ciencia y la Cultura (OEI) entre 2004 y 2012. Además, fue Secretario de Estado de Educación y Director General de Educación del Ministerio de Educación de España desde 1986 a 1996. En 1994 lideró junto con la UNESCO la realización de la Conferencia Mundial de Salamanca. Ha publicado numerosos libros, capítulos de libros y artículos científicos, sobre diversas temáticas vinculadas a la educación, siendo sus áreas de interés la calidad y equidad de la educación, la inclusión educativa y atención a la diversidad. ORCID ID: https://orcid.org/oooooo01-9429-7674. Email: amarches@psi.ucm.es

\section{Laura Hernández}

Doctora en educación por la Universidad Complutense Madrid, Licenciada en Psicología. Profesora asociada de la facultad de psicología de la Universidad Complutense de Madrid. Además, ejerce como orientadora especializada en atención a la diversidad, educación infantil y neuropsicología en la Comunidad de Madrid. Entre 2010 y 2014, desempeñó el cargo de Técnico de programas de la Dirección General de Programas Educativos y 
A. Marchesi y L. Hernández

Culturales en la Organización de Estados Iberoamericanos para la Educación, la Ciencia y la Cultura (OEI). ORCID ID: https://orcid.org/o0o0-0003-0434-679X. Email:

laurhern@ucm.com 